\title{
Development of a Full-Thickness Human Skin Equivalent In Vitro Model Derived from TERT-Immortalized Keratinocytes and Fibroblasts
}

\author{
Christianne M.A. Reijnders, PhD, Amanda van Lier, MSc, Sanne Roffel, Ing, ${ }^{1}$ \\ Duco Kramer, Ing, ${ }^{2}$ Rik J. Scheper, $\mathrm{PhD}^{3}$ and Susan Gibbs, $\mathrm{PhD}^{1,4}$
}

Currently, human skin equivalents (HSEs) used for in vitro assays (e.g., for wound healing) make use of primary human skin cells. Limitations of primary keratinocytes and fibroblasts include availability of donor skin and donor variation. The use of physiologically relevant cell lines could solve these limitations. The aim was to develop a fully differentiated HSE constructed entirely from human skin cell lines, which could be applied for in vitro wound-healing assays. Skin equivalents were constructed from human TERT-immortalized keratinocytes and fibroblasts (TERT-HSE) and compared with native skin and primary HSEs. HSEs were characterized by hematoxylin-eosin and immunohistochemical stainings with markers for epidermal proliferation and differentiation, basement membrane $(\mathrm{BM})$, fibroblasts, and the extracellular matrix (ECM). Ultrastructure was determined with electron microscopy. To test the functionality of the TERT-HSE, burn and cold injuries were applied, followed by immunohistochemical stainings, measurement of reepithelialization, and determination of secreted wound-healing mediators. The TERT-HSE was composed of a fully differentiated epidermis and a fibroblast-populated dermis comparable to native skin and primary HSE. The epidermis consisted of proliferating keratinocytes within the basal layer, followed by multiple spinous layers, a granular layer, and cornified layers. Within the TERT-HSE, the membrane junctions such as corneosomes, desmosomes, and hemidesmosomes were well developed as shown by ultrastructure pictures. Furthermore, the BM consisted of a lamina lucida and lamina densa comparable to native skin. The dermal matrix of the TERT-HSE was more similar to native skin than the primary construct, since collagen III, an ECM marker, was present in TERT-HSEs and absent in primary HSEs. After wounding, the TERT-HSE was able to reepithelialize and secrete inflammatory wound-healing mediators. In conclusion, the novel TERT-HSE, constructed entirely from human cell lines, provides an excellent opportunity to study in vitro skin biology and can also be used for drug targeting and testing new therapeutics, and ultimately, for incorporating into skin-on-a chip in the future.

Introduction

$\mathbf{H}$ UMAN SKIN EQUIVALENTS (HSEs) are important models for fundamental research, for industry purposes (cytotoxicity studies, drug targeting, testing new therapeutics, and treatment strategies) and for clinical applications. The need for physiologically relevant HSE in vitro models is increasing, since the EU regulations encourage replacement, reduction, and refinement of animal models (EU Directive 2010/63/EU) and since a ban was introduced for testing cosmetic ingredients in animals (EU Cosmetic Directive 76/768/EEC; EU Cosmetics Products Regulation [EC] No1223/2009; REACH Regulation [EC] 1907/2006). Therefore, HSE in vitro models are not only indispensable for classification and risk assessment studies of chemicals (e.g., cytotoxicity, irritancy) ${ }^{1-5}$ but they also offer a unique model to study normal and abnormal skin biology, including wound healing, skin disease, and infection. Examples of disease models include abnormal scar

\footnotetext{
${ }^{1}$ Department of Dermatology, VU University Medical Centre, Amsterdam, The Netherlands.

${ }^{2}$ Department of Dermatology, University Medical Centre Groningen, Groningen, The Netherlands.

${ }^{3}$ Department of Pathology, VU University Medical Centre, Amsterdam, The Netherlands.

${ }^{4}$ Department of Oral Cell Biology, Academic Centre for Dentistry Amsterdam (ACTA), University of Amsterdam and VU University Amsterdam, Amsterdam, The Netherlands.

(C) C.M.A. Reijnders 2015; Published by Mary Ann Liebert, Inc. This Open Access article is distributed under the terms of the Creative Commons Attribution Noncommercial License (http://creativecommons.org/licenses/by-nc/4.0/) which permits any noncommercial use, distribution, and reproduction in any medium, provided the original author(s) and the source are credited.
} 
formation (e.g., keloid), melanoma invasion, psoriasis, and skin blistering. ${ }^{3,6-13}$ HSEs are used for bacterial adhesion and infection studies. ${ }^{12,14,15}$ In addition, HSEs are suitable for investigation of the effects of chemotherapeutics, drug delivery of pharmaceuticals, photoprotective properties of various compounds, and the xenobiotic metabolism. ${ }^{3,16-22}$

Developments over the last 30 years have led to HSEs being constructed from primary cells, which very closely resemble native skin. The epidermis, being the outermost layer of the skin, forms an important barrier to pathogens and prevents dehydration. Renewal of the epidermis is a continuous, tightly regulated differentiation process. In HSEs, similar to native skin, proliferation is strictly regulated by the keratinocytes of the basal layer. In native human skin, the proliferation rate within the stratum basale (SB) is $10-12 \%$, as shown by the Ki67 protein expression. ${ }^{23}$ When keratinocytes make a commitment to terminally differentiate, they migrate from the basal layer to form the suprabasal layers. ${ }^{23-26}$ The epidermal cells undergo several morphological and biochemical changes leading to the following structural layers: the basal layer, spinous layer, granular layer, and cornified layer. Each epidermal layer is characterized by the production of their specific epidermal differentiation proteins. The cuboidal-shaped cells of SB express keratin 5 and 14 (K5/K14), whereas the suprabasal spinous layers produce keratin 1 and keratin 10 (K1/K10). The epidermal cells in the stratum granulosum (SG) stop to synthesize keratins and start with the production of late epidermal differentiation proteins (e.g., involucrin, loricrin, and filaggrin). The final stage of keratinocyte terminal differentiation involves formation of the cornified envelope known as the stratum corneum (SC). When the skin becomes damaged or during skin disease (e.g., wounding, psoriasis), the expression of a stress-related hyperproliferative marker, keratin 6 (K6), is increased in all layers of the epidermis. ${ }^{23,27,28}$ Hypertrophic scar formation is associated with increased alpha-smooth muscle actin ( $\alpha$-SMA) expression. ${ }^{10}$

The membrane junctions, which interconnect keratinocytes within the SB, stratum spinosum (SS), and SG, are called desmosomes. ${ }^{29}$ At the interface of the SG and SC, desmosomes transform into corneosomes. ${ }^{30}$ Malformation of desmosomes might lead to blistering diseases. ${ }^{31-33}$

The epidermis is attached to the basement membrane (BM), which is situated between the epidermis and dermis. The BM can be divided into a lamina lucida and lamina densa. It anchors the epithelial cells to the dermal matrix with hemidesmosomes and functions as a mechanical barrier. The hallmark of the BM is the expression of laminin 5 and collagen IV. ${ }^{34}$ The dermis consists mainly of connective tissue and is responsible for elasticity and tensile strength of the skin. The main cell type found within the dermis is the spindle-shaped fibroblast, which is positive for the mesenchymal vimentin marker and communicates extensively with keratinocytes within the epidermis, leading to synthesis of the extracellular BM and dermal matrix. The dermal extracellular matrix (ECM) is predominantly characterized by elastin, fibronectin, and multiple types of collagen. Maintenance and restoration of the skin homeostasis is also established by interaction between the dermal fibroblasts and epidermal keratinocytes, which secrete a cocktail of cytokines, chemokines, and growth factors. $9,35-38$

The currently existing HSEs are generally constructed of primary human skin cells, which originate from freshly isolated healthy human skin obtained from standard surgical procedures. The production is limited by the following: (i) logistical issues concerning restricted supply of fresh, healthy donor skin, (ii) donor variation, and (iii) restricted proliferation and amplification capacity of primary cells. The use of physiologically relevant human skin cell lines, for example, hTERT-immortalized keratinocytes and fibroblasts, will overcome these limitations and provide the possibility for higher throughput screening of new products and new drugs. However, it should be realized that donor variation is sometimes required to accurately resemble the population and this should not be forgotten when using cell lines. In addition, the ethical issues for obtaining the donor tissue for modification still exist, but are now limited to a single donor, rather than many donors, due to immortalization of the cells. In recent years, several researchers have developed HSEs, which incorporate keratinocyte cell lines for the epidermal component; however, for the dermal compartment, they used human primary fibroblasts, human fetal lung fibroblasts (MRC5), or 3T3 mouse fibroblasts. ${ }^{39-53}$ At the moment, to the best of our knowledge, there are no full-thickness skin equivalents (SEs) available, which completely consist entirely out of physiologically relevant human skin cell lines.

Immortalization with hTERT cDNA will overcome cell senescence of primary cells. During each normal cell division, the telomeres of primary cells are shortened. The exogenous hTERT cDNA, which encodes for the catalytic subunit of human telomerase, prevents telomere shortening and, thereby, increases the life span of the cells, while maintaining the normal physiology and phenotypic properties of the cells. ${ }^{54,55}$

In our study, we determined whether hTERT-immortalized human newborn foreskin keratinocytes with spontaneous loss of p16 expression (N/TERT-1) ${ }^{50}$ are suitable for reconstructing a fully differentiated epidermis in combination with hTERT-immortalized human newborn foreskin fibroblasts (BJ-5ta), which are incorporated into the dermal compartment of the SE. Both cell lines retained their normal morphology and proliferation rate in the two-dimensional culture. $^{50,56}$ The aim of our study was to develop a fully differentiated HSE constructed entirely from human skin TERT-immortalized cell lines, which resembles native human skin and would be applicable for a broad range of in vitro skin studies. The TERT-HSE was intensively characterized at the histological level (hematoxylin and eosin $[\mathrm{H} \& \mathrm{E}]$ staining and immunohistochemistry of epidermal and dermal markers) and ultrastructural level (electron microscopy $[\mathrm{EM}])$. In addition, the protein secretome (cytokines, chemokines, and growth factors) was analyzed by ELISA. Finally, as proof-of-concept for functionality, the TERT-HSE was compared with our in vitro wound-healing model, in which primary cells are currently used. ${ }^{9}$

\section{Materials and Methods}

\section{Cell culture of human primary cells and TERT-immortalized keratinocytes and fibroblasts}

Primary human epidermal keratinocytes were isolated from neonatal foreskin as described earlier. ${ }^{57}$ Both primary keratinocytes and N/TERT-1 keratinocytes ${ }^{50}$ were cultured in Dulbecco's modified Eagle's medium (DMEM)/Ham's F12 (3:1) (Gibco), $50 \mu \mathrm{g} / \mathrm{mL}$ gentamycin (Centrafarm), 1\% UltroserG (UG) (Bio Sepra SA), $1 \mu \mathrm{M}$ hydrocortisone, 
$0.1 \mu \mathrm{M}$ insulin, $1 \mu \mathrm{M}$ isoproterenol, $2 \mathrm{ng} / \mathrm{mL} \mathrm{KGF}$ at $37^{\circ} \mathrm{C}$, and $7.5 \% \mathrm{CO}_{2}$ (primary) or $5 \% \mathrm{CO}_{2}$ (cell line). The culture medium was renewed twice per week. Cultures with 70 $80 \%$ confluency were used for the construction of HSEs. N/TERT-1 was provided by Rheinwald and the Cell Culture Core of Harvard Skin Disease Research Center.

Primary human dermal fibroblasts were isolated from neonatal foreskin as described earlier. ${ }^{58}$ Both primary dermal fibroblasts and hTERT BJ-5ta fibroblasts (ATCC, LGC Standards $\mathrm{GmbH}$ ) were cultured within DMEM (Gibco), $1 \%$ $\mathrm{UG}$, and an antibiotic $(50 \mu \mathrm{g} / \mathrm{mL}$ gentamycin for primary fibroblasts and $0.01 \mathrm{mg} / \mathrm{mL}$ hygromycin for hTERT BJ-5ta fibroblasts) at $37^{\circ} \mathrm{C}$ and $5 \% \mathrm{CO}_{2}$. Culture medium was renewed twice per week. Cultures with $70-80 \%$ confluency were used for the construction of HSEs. Culture additives were obtained from Sigma-Aldrich, unless otherwise stated.

\section{Human skin equivalent}

The HSE was constructed as described earlier. ${ }^{10}$ Briefly, the primary or cell line fibroblasts $\left(4 \times 10^{5}\right.$ cells $)$ were seeded onto a bovine matrix without a BM $\left(2.2 \times 2.2 \mathrm{~cm}^{2}\right)$ (MatriDerm, Med Skin Solutions Dr. Suwelack AG). This matrix consists of collagen types I, III, and V, and elastin. It does not show cross-reactivity with human IHC antibodies, except for collagen I and elastin. These fibroblast sheets were cultured submerged for 3 weeks in the fibroblast sheet medium (DMEM/Ham's F12 [3:1], $50 \mu \mathrm{g} / \mathrm{mL}$ gentamycin, 2\% UG, $5 \mu \mathrm{g} / \mathrm{mL}$ insulin, $50 \mu \mathrm{g} / \mathrm{mL}$ ascorbic acid (Sigma), and $5 \mathrm{ng} /$ $\mathrm{mL}$ epidermal growth factor). After 3 weeks, the primary or cell line keratinocytes $\left(5 \times 10^{5}\right.$ cells $)$ were seeded onto the matrices populated with the corresponding primary or cell line fibroblasts. After $4 \mathrm{~h}$ of attachment, the cultures were placed submerged for 3-4 days within DMEM/Ham's F-12 (3:1), $1 \% \mathrm{UG}, 50 \mu \mathrm{g} / \mathrm{mL}$ gentamycin, $1 \mu \mathrm{M}$ hydrocortisone, $1 \mu \mathrm{M}$ isoproterenol, $0.1 \mu \mathrm{M}$ insulin, and $2 \mathrm{ng} / \mathrm{mL} \mathrm{KGF}$; thereafter, the cultures were placed air-exposed for 14 days in DMEM/Ham's F-12 (3:1), 0.2\% UG, $50 \mu \mathrm{g} / \mathrm{mL}$ gentamycin, $1 \mu \mathrm{M}$ hydrocortisone, $1 \mu \mathrm{M}$ isoproterenol, $0.1 \mu \mathrm{M}$ insulin, $10 \mu \mathrm{M}$ L-carnitine, $10 \mathrm{mM}$ L-serine, $1 \mu \mathrm{M}$ DL- $\alpha$-tocopherol acetate, and enriched with a lipid supplement containing $25 \mu \mathrm{M}$ palmitic acid, $15 \mu \mathrm{M}$ linoleic acid, $7 \mu \mathrm{M}$ arachidonic acid, and $24 \mu \mathrm{M}$ bovine serum albumin. The culture medium was refreshed twice a week. Finally, the HSEs were harvested for histological analysis (H\&E, IHC, and EM), and a 24-h supernatant was collected for ELISA.

\section{Burn and cold injuries}

Burn injury or cold injury was applied to the TERT-HSEs after $\sim 2$ weeks of air-exposed culture as described previously. ${ }^{9}$ Times and temperatures for obtaining full-thickness injury were optimized by heating cultures for different durations and time periods followed by histological examination 1 day after injury before finalizing the protocol (data not shown). For full-thickness burn injury, a metal device (tip with a length of $2 \mathrm{~cm}$ and a width of $2 \mathrm{~mm}$ ) attached to a Weller soldering station (WSD81 Cooper Tools), heated continuously at $117^{\circ} \mathrm{C}$, was applied for $10 \mathrm{~s}$ to the SC of the TERT-HSEs. Cold injuries were applied with a metal device with similar dimensions as the burn injury device. This device was cooled down to $-196^{\circ} \mathrm{C}$ in liquid nitrogen and directly applied with gentle pressure to the SC for $10 \mathrm{~s}$. After wounding, TERT-HSEs continued air-exposed culture. Wound closure and soluble mediator secretion were analyzed at day 1, 3, and 7 after wounding. HSEs were given new culture media $24 \mathrm{~h}$ before collecting the supernatant for soluble mediator analysis at each time interval.

\section{Morphology, immunohistochemistry, and ultrastructure}

For histological analysis, the HSEs of three independent experiments (e.g., primary cells: three different donors; cell lines: three different passages) were fixed within $4 \%$ formaldehyde and embedded in paraffin. Paraffin sections $(5 \mu \mathrm{m})$ were cut and used for H\&E or IHC staining. The antibodies for IHC are summarized in Table 1 and were used in combination with EnVision (Dako) followed by the AEC substrate to visualize the specific antibody. Brightfield photographs (100- and 200-fold magnification) were made with a Nikon microscope, a digital camera, and NIS

Table 1. Primary Antibodies Used for Immunohistochemical Staining

\begin{tabular}{|c|c|c|c|c|c|}
\hline Antibody & Dilution & Isotype & Material & Antigen retrieval & Company \\
\hline Collagen IV & $1: 20$ & $\operatorname{IgG} 2 b$ & Paraffin & Citrate buffer, ${ }^{\mathrm{a}}$ Pepsin ${ }^{\mathrm{b}}$ & Monosan \\
\hline Involucrin & $1: 1200$ & IgG1 & Paraffin & - & Novocastra \\
\hline Keratin 5 & $1: 150$ & IgG1 & Paraffin & Citrate buffer, ${ }^{\mathrm{a}}$ Pepsin $^{\mathrm{b}}$ & Monosan \\
\hline Keratin 6 & $1: 150$ & IgG1 & Paraffin & Citrate buffer ${ }^{a}$ & Progen Biotechnik GmbH \\
\hline Keratin 10 & $1: 500$ & IgG1 & Paraffin & Citrate buffer, ${ }^{\mathrm{a}}$ Pepsin ${ }^{\mathrm{b}}$ & ICN Biomedicals \\
\hline Ki67 & $1: 50$ & IgG1 & Paraffin & Citrate buffer ${ }^{c}$ & Dako \\
\hline Loricrin & $1: 1200$ & Rabbit serum & Paraffin & - & Covance \\
\hline Vimentin & $1: 200$ & IgG1 & Paraffin & Citrate buffer ${ }^{a}$ & Dako \\
\hline Collagen III & $1: 2400$ & IgG1 & Paraffin & Citrate buffer, ${ }^{\mathrm{a}}$ Pepsin ${ }^{\mathrm{b}}$ & Abcam \\
\hline Fibronectin & $1: 1000$ & IgG1 & Frozen & - & Santa-Cruz Biotechnology \\
\hline Laminin 5 & $1: 400$ & IgG2a & Frozen & - & Dako \\
\hline $\begin{array}{l}\text { Alpha-smooth } \\
\text { muscle actin }\end{array}$ & $1: 4000$ & IgG2a & Paraffin & - & Dako \\
\hline
\end{tabular}

\footnotetext{
${ }^{\mathrm{a}}$ The slides were heated at $100^{\circ} \mathrm{C}$ in $0.01 \mathrm{M}$ citrate buffer $(\mathrm{pH}$ 6.0) in a microwave and cooled to room temperature for at least $2 \mathrm{~h}$ before adding the antibody.

${ }^{\mathrm{b}}$ After antigen retrieval, the slides were incubated with $4 \mathrm{mg} / \mathrm{mL}$ pepsin in $0.2 \mathrm{~N} \mathrm{HCl}$ for $15 \mathrm{~min}$.

${ }^{\mathrm{c}}$ The slides were heated at $100^{\circ} \mathrm{C}$ in $0.01 \mathrm{M}$ citrate buffer $(\mathrm{pH}$ 6.0) on a cooker for $30 \mathrm{~min}$ and cooled to room temperature for at least $2 \mathrm{~h}$ before adding the antibody.
} 
Elements AR version 2.10 software (Nikon Instruments Europe B.V.). The proliferation index is expressed as the number of Ki67 positively stained nuclei divided by the total number of basal epidermal cells $\times 100 \%$, as described earlier. ${ }^{23}$ Reepithelialization was defined as the distance that the newly formed epidermis had migrated into the wound bed and was measured with NIS Elements.

The ultrastructure was determined with EM. Native skin, primary HSEs, and TERT-HSEs were regularly fixed in $4 \%$ glutaraldehyde in $0.1 \mathrm{M}$ sodium cacodylate $\mathrm{pH} 7.4$, postfixated with $1 \%$ osmium tetroxide in $1.5 \%$ potassium ferricyanide $\left(\mathrm{K}_{3}\left[\mathrm{Fe}(\mathrm{CN})_{6}\right]\right)$ in $0.1 \mathrm{M}$ sodium cacodylate $\mathrm{pH} 7.4$, and embedded in the EPON resin. The 70-nm sections were imaged at the Philips CM100 EM or the Zeiss Supra 55 EM.

\section{Enzyme-linked immunosorbent assay}

Protein secretion levels within the 24-h supernatant samples collected from primary HSEs and TERT-HSEs were determined with the enzyme-linked immunosorbent assay (ELISA), as described earlier by Spiekstra et al. ${ }^{37}$ Results are expressed as $\mathrm{pg} / \mathrm{mL} / 24 \mathrm{~h}$ or $\mathrm{ng} / \mathrm{mL} / 24 \mathrm{~h}$. ELISA reagents were used for chemokine, cytokine, and growth factor quantification in accordance to the manufacturer's specifications. All antibodies were supplied by R\&D Systems, Inc., except for CXCL-8/IL-8 and TNF- $\alpha$, PeliPair reagent sets, supplied by Sanquin.

\section{Statistics}

Statistical analysis was performed with the aid of GraphPad Prism, version 6 (GraphPad Software, Inc.). Specific details of the statistic tests are mentioned in the legends. Differences were considered significant when $p<0.05$.

\section{Results}

\section{Human TERT-immortalized cell line SE resembles native skin}

Histological analysis demonstrated that the TERT-HSEs retained the morphological characteristics of normal, native human skin and were comparable to our in-house primary HSEs. The TERT-HSEs showed a well-defined epidermis on top of a fibroblast-populated matrix. The epidermis consisted of an SB, a multilayered SS, SG, and SC (Fig. 1). The Ki67 proliferation index of the cells in the SB of TERT-
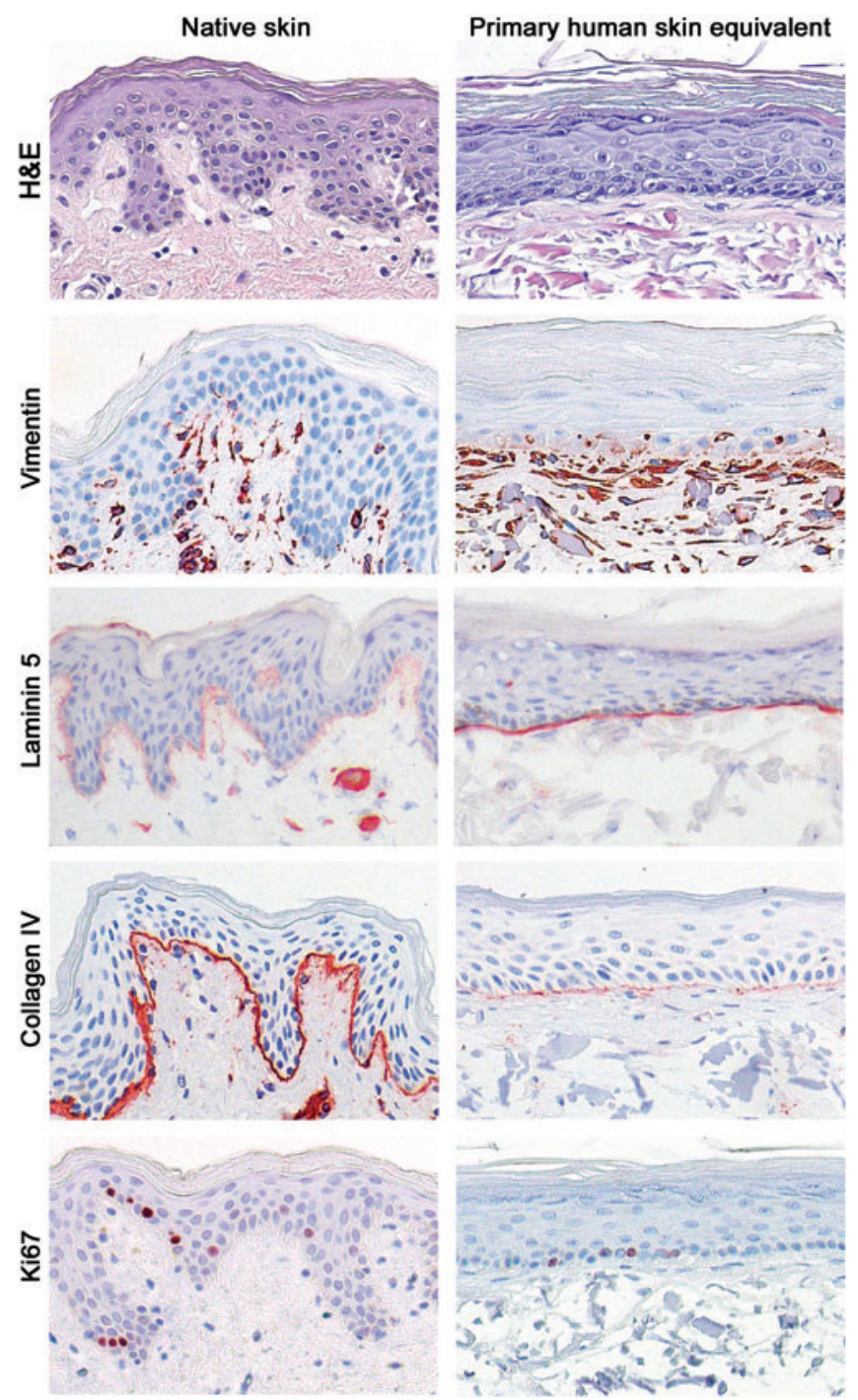

\section{Cell line human skin equivalent}
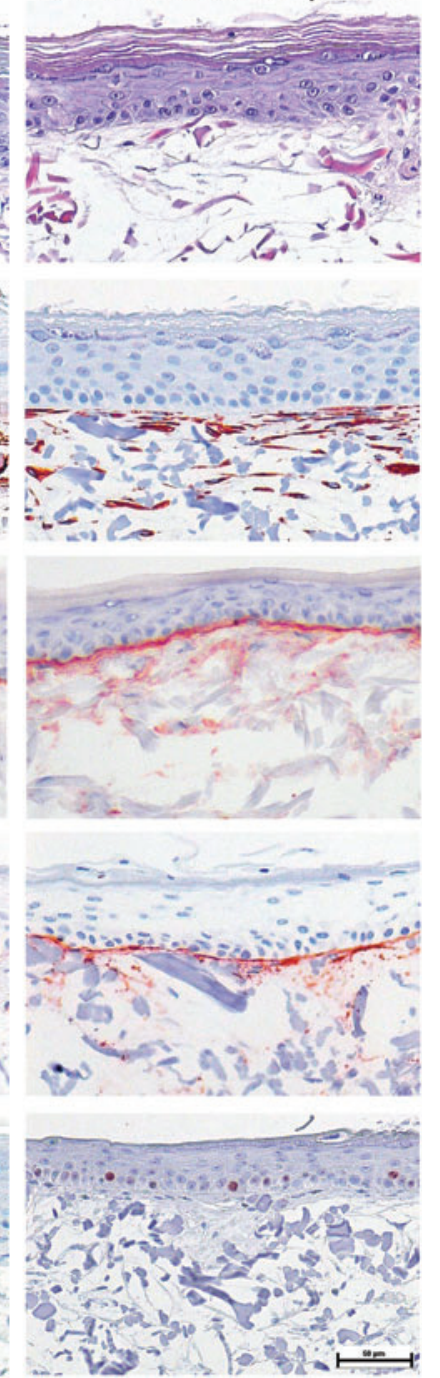

FIG. 1. Histological appearance of primary and TERT-immortalized cell line SEs resembles native skin. Characterization of 2-week air-exposed reconstructed HSEs, which were developed with human primary or human TERT-immortalized keratinocytes and fibroblasts. Histology of native skin and the different SEs is shown with a hematoxylin and eosin staining and immunohistochemical stainings using antibodies directed against human vimentin (fibroblasts), laminin 5, collagen IV (basement membrane: lamina lucida and lamina densa, respectively), and Ki67 (proliferation index). Stainings were performed on representative HSEs derived from three independent culture experiments. Scale bar represents $50 \mu \mathrm{m}$. HSEs, human skin equivalents; SEs, skin equivalents. Color images available online at www .liebertpub.com/tea 
HSEs $(14.5 \% \pm 2.7 \%$, mean $\pm \mathrm{SD}, n=7)$ was comparable to primary HSEs $(13.5 \% \pm 4.4 \%$, mean $\pm \mathrm{SD}, n=7)$ (Fig. 1) and closely resembled our previously reported proliferation rate of native skin $(10-12 \%) .{ }^{23}$ The TERT-HSEs as well as primary HSEs showed vimentin-positive fibroblasts in the dermis similar to native skin (Fig. 1). As in native skin, the TERT-HSEs showed expression of the BM proteins, laminin 5 (lamina lucida) and collagen IV (lamina densa), which were located at the interface of the collagen-elastin matrix and the epidermis. The collagen IV expression was more pronounced in TERT-HSEs compared to primary HSEs (Fig.
1). The BM markers were absent in the original collagenelastin matrix, which was used as the dermal component (data not shown).

Next, the protein expression of epidermal differentiation markers was examined. As in native skin and primary HSEs, K5 was predominantly located within the cuboidal epidermal cells of the SB and lower spinous layers and K10 was expressed in all suprabasal epidermal layers of the TERT-HSEs (Fig. 2). Positive IHC staining for the cornified envelope precursor involucrin was observed in the upper layers of the SS and predominantly in the SG,
FIG. 2. Epidermal differentiation and dermal matrix markers within 2-week airexposed primary and cell line SEs. Representative immunohistochemical stainings for epidermal differentiation and dermal matrix markers on native skin, primary, and cell line HSE sections are shown. Markers represent different stages of epidermal differentiation: for example, early (keratin 5), intermediate (keratin 10 and involucrin), and late (loricrin) epidermal differentiation. Keratin 6 is a hyperproliferative marker. Collagen III is a component of the dermal ECM. Alphasmooth muscle actin is a myofibroblast marker and is associated with scar formation. Stainings were performed on representative HSEs derived from three independent culture experiments. Scale bar represents $50 \mu \mathrm{m}$ (upper epidermal panels) or $100 \mu \mathrm{m}$ (dermal panels). ECM, extracellular matrix. Color images available online at www .liebertpub.com/tea
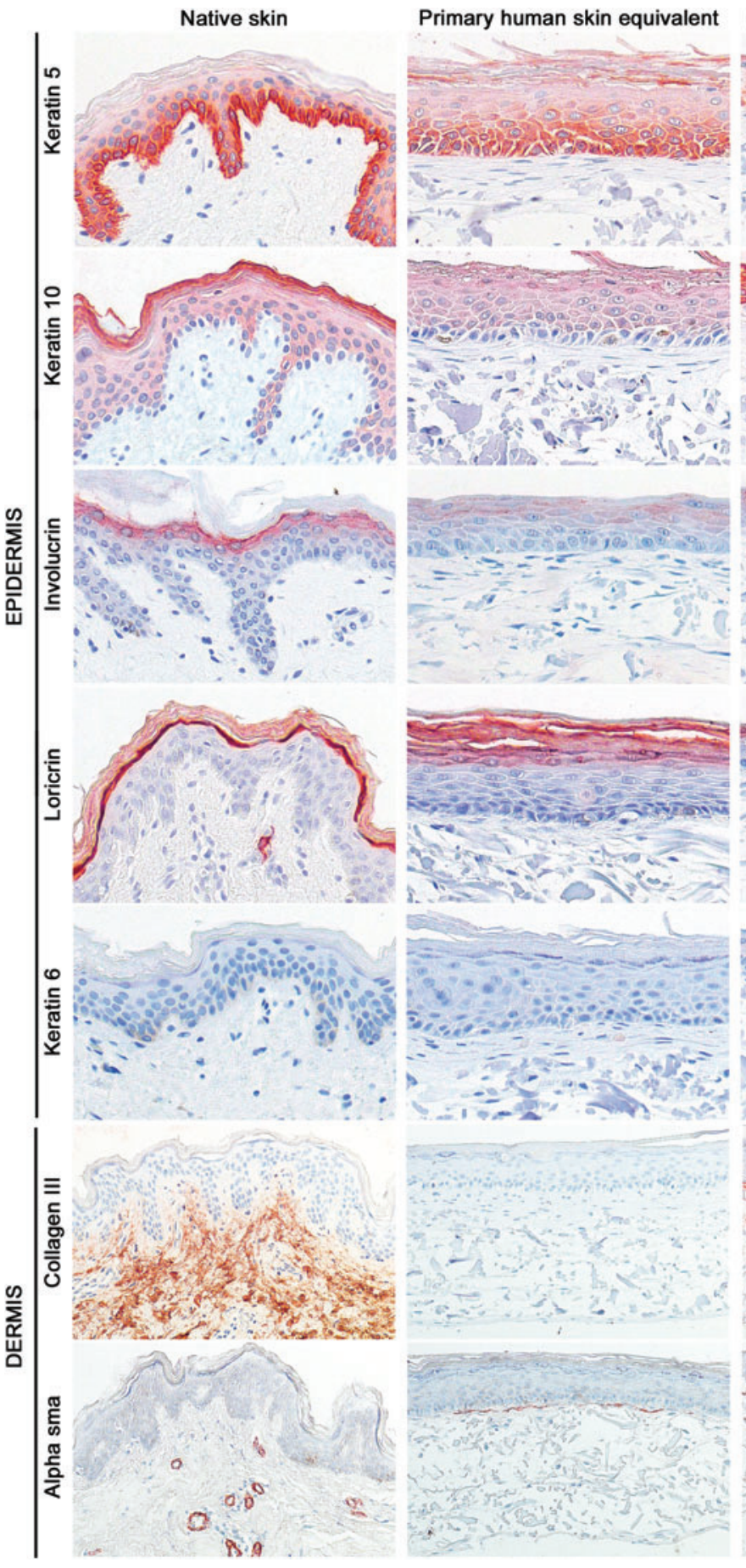

Cell line human skin equivalent
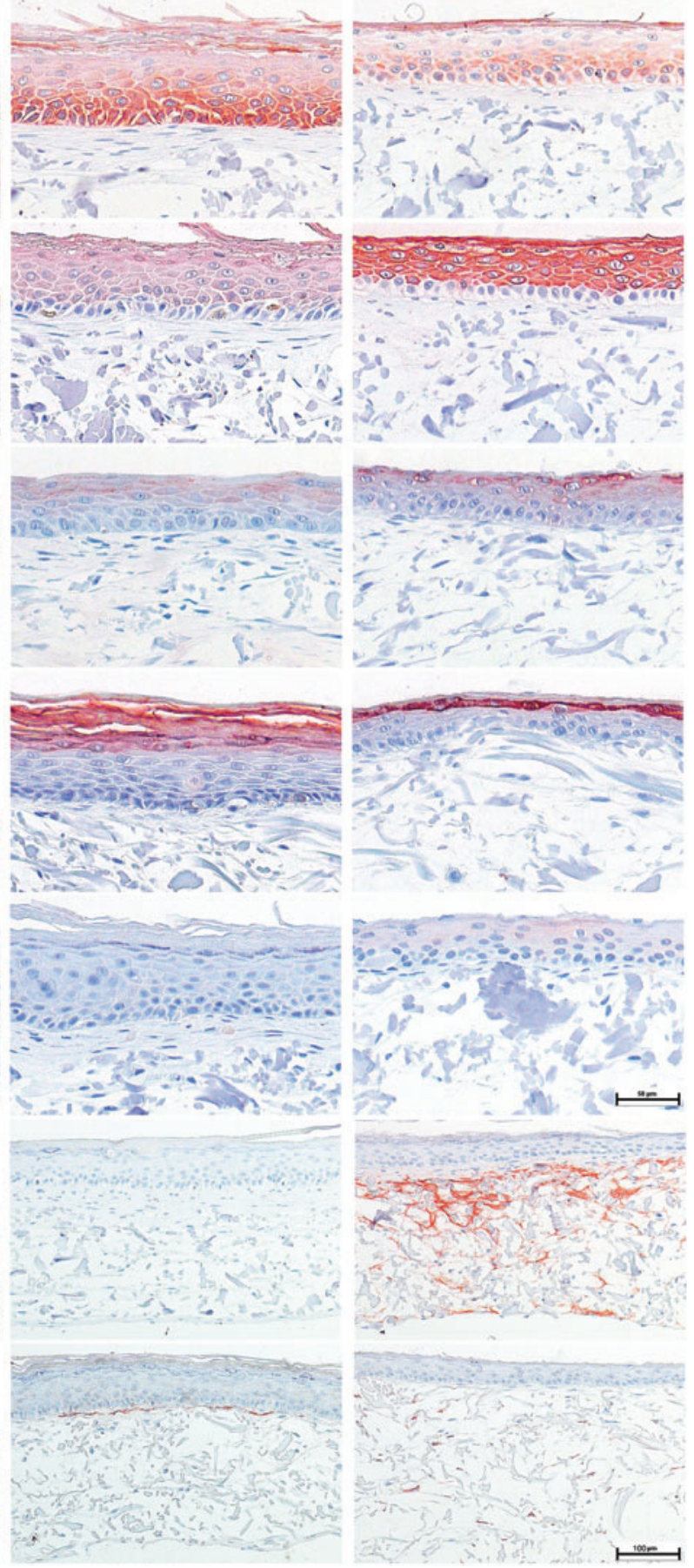
and loricrin, the late terminal differentiation marker, was observed in the SG of the TERT-HSEs, which was comparable with the expression pattern of involucrin and loricrin within native skin as well as primary HSEs (Fig. 2). The hyperproliferative marker K6 was absent in native skin and primary and TERT-HSEs (Fig. 2).

In addition, collagen III, a native ECM marker, was expressed within the dermal compartment of TERT-HSEs with the TERT fibroblasts, whereas it was absent in primary HSEs (Fig. 2). However, fibronectin, another ECM marker, was present in the dermis of both primary as well as TERT-
HSEs (data not shown). Intermittent $\alpha$-SMA staining, representing sporadic myofibroblasts, was randomly present in the dermal matrix of TERT-HSEs, whereas it was located directly underneath the BM within primary HSEs (Fig. 2).

The EM pictures showed that the ultrastructure of TERTHSEs was comparable to native skin (Fig. 3) and primary HSEs (not shown). The TERT-HSEs presented well-stratified epithelial layers on top of a fibroblast-populated dermal matrix with collagen bundles confirming the H\&E and IHC results. The SC consisted of multiple cornified layers connected with corneosomes. The SG and SS presented multiple
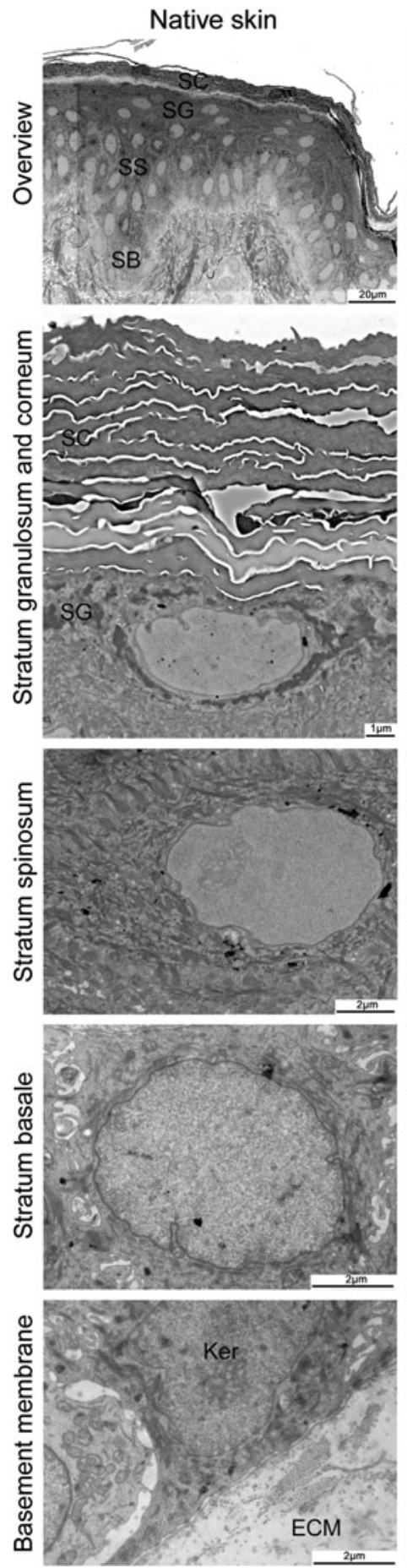

\section{Cell line human skin equivalent}
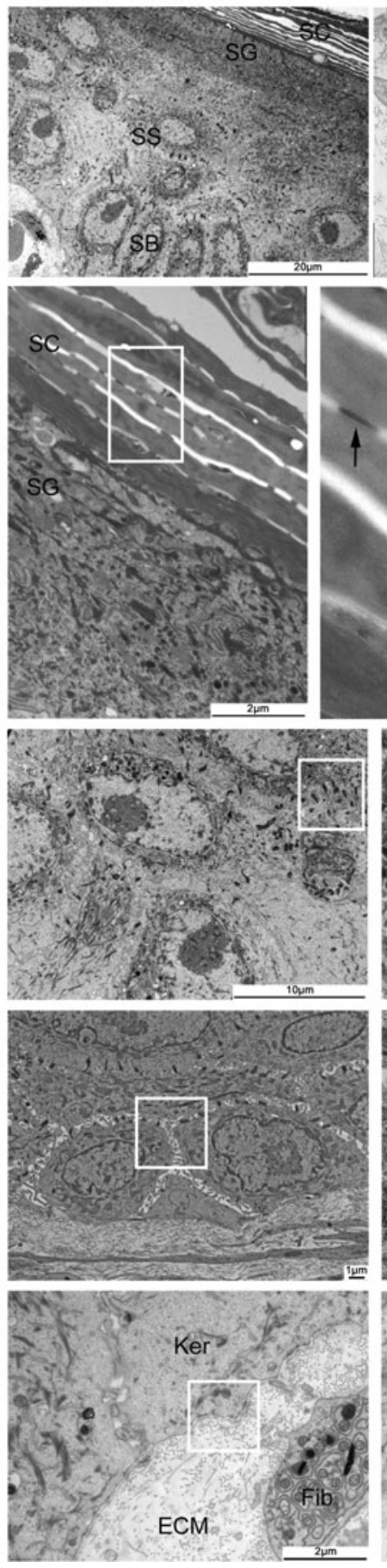
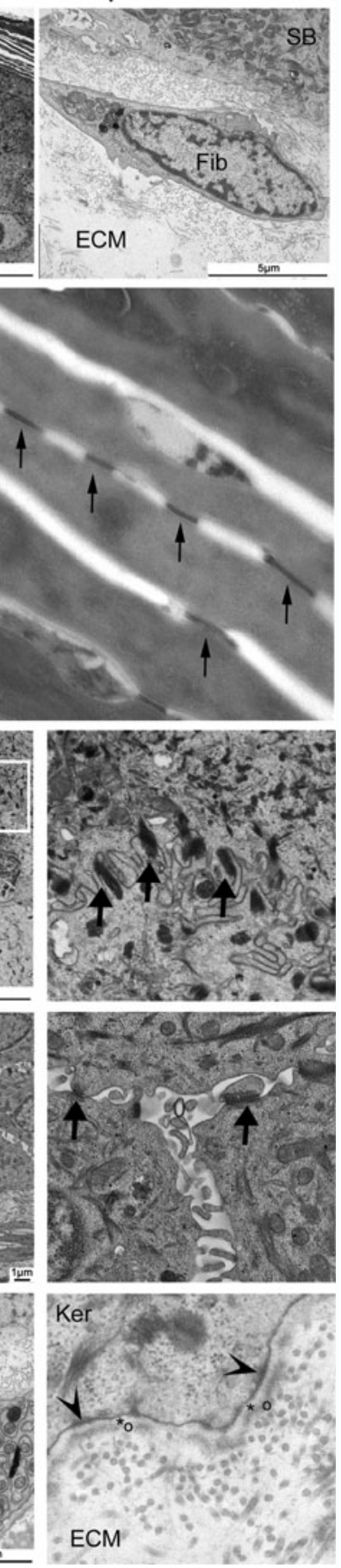

FIG. 3. Ultrastructure of TERT-immortalized cell line SEs resembles native skin. Ultrastructure pictures of native skin (left column) and cell line HSE (middle and right column [detail of white square; fourfold]). EM overview picture of the epidermis of native skin and TERTHSE. Fibroblasts are located in between the collagen bundles of the dermal matrix (ECM). Corneosomes (small arrows) are present within the SC and at the SG/SC interface. Desmosomes (large arrows) are depicted within the SG, SS, and SB. Hemidesmosomes (arrow heads) at the bottom of the SB attaching the epidermis to dermis.

Basement membrane contains a lamina lucida $(*)$ and lamina densa $(O)$. SC, stratum corneum; SG, stratum granulosum; SS, stratum spinosum; $\mathrm{SB}$, stratum basale; Ker, keratinocyte; Fib, fibroblast. 
desmosomes within the cell membrane of the keratinocytes. The intracellular space between the keratinocytes within the SS was smaller compared to the intracellular space of the cuboidal keratinocytes in the SB. Multiple hemidesmosomes were present at the lower side of the SB and anchored the epidermal cells to the ECM. The BM clearly showed a lamina lucida and lamina densa (Fig. 3), developed within 2 weeks of air-exposed culture time. Along the BM we observed some exocytose-like vacuoles in both cell line and primary HSEs, and native skin. The presence of mitochondria referred to a healthy status of the tissue.

In conclusion, extensive morphological characterization shows that the HSE constructed entirely from the TERTimmortalized human skin keratinocyte and fibroblast cell lines closely resembles normal, native human skin. A fully differentiated epidermis is well formed on a fibroblastpopulated dermis, which is very similar to our HSE constructed from primary skin cells that are not immortalized.

\section{Human TERT-immortalized cell line SE secretes cytokines and chemokines}

Since the skin continuously secretes basal levels of cytokines, chemokines, and growth factors to maintain skin homeostasis, we determined the basal secretion of these factors within the culture supernatant of the TERT-HSE during a 24-h period and compared these secretion profiles with the secretome of freshly excised ex vivo skin $^{37}$ and primary HSEs. Three groups of proteins could be distinguished by ELISA (Table 2). In the first group (TERT$\mathrm{HSE}=$ ex vivo skin), chemokines, CXCL-8/IL-8 and CCL-5/ RANTES, and TIMP-2 secretion from TERT-HSEs were similar to ex vivo skin. Cytokines, TNF- $\alpha$ and IL- $1 \alpha$, were low or undetectable in both. In the second group (TERTHSE $>$ ex vivo skin), inflammatory/angiogenic mediator secretion of CXCL-1/GRO- $\alpha$ and CCL-2/MCP-1 was more prominent within TERT-HSEs compared to ex vivo skin. In the third group (TERT-HSE $<$ ex vivo skin), cytokine IL-6 and growth factor, VEGF and HGF, secretion was more abundant in the supernatant of ex vivo skin compared to the TERT-HSE supernatant. Indeed, VEGF and HGF secretion was below the detection limit of the ELISA in TERT-HSEs.

Comparison of the primary HSE protein profiles to the ex vivo skin protein profiles resulted in a similar secretion profile as the TERT-HSE versus ex vivo skin, except for CXCL-8/IL-8 and CCL-5/RANTES secretion, which was similar between TERT-HSE and ex vivo skin. However, CXCL-8/IL-8 and CCL-5/RANTES secretion was significantly increased within primary HSEs compared to ex vivo skin (Table 2). Furthermore, when comparing primary HSEs with TERT-HSEs, secretion of CXCL-1/GRO- $\alpha$, CCL2/MCP-1, VEGF, and HGF was more abundant in primary HSEs compared to TERT-HSEs (Table 2). In conclusion, the TERT-HSE is able to secrete a broad panel of cytokines and chemokines, which is more characteristic for adult ex vivo skin than primary foreskin HSE obtained from juveniles.

\section{Human TERT-immortalized cell line SE shows inflammatory cytokine and chemokine secretion during wound closure}

Previously, we have shown that primary HSEs can be used to study inflammatory mediator release during wound healing. ${ }^{9}$ Therefore, burn and cold injuries were made within the TERT-HSE cultures to test the wound-healing capacity of the immortalized cell line three-dimensional (3D) model. Reepithelialization and secretion of (pro)inflammatory mediators into the supernatant were investigated at 1,3, and 7 days after wounding. Wound healing was already apparent 1 day after injury, with reepithelialization being slightly more enhanced 1 day after cold injury compared to burn injury (Fig. $4 \mathrm{~A}, \mathrm{~B})$. Ingrowth after wounding was a continuous process with almost complete reepithelialization resulting in a welldifferentiated epidermis after 7 days (Fig. 4C, D).

Table 2. Basal Levels of Wound-Healing Mediator Secretion Under Homeostatic Conditions (PG/ML/24 H)

\begin{tabular}{|c|c|c|c|c|}
\hline Protein $(\mathrm{pg} / \mathrm{mL} / 24 \mathrm{~h})$ & Function & $\begin{array}{c}T E R T-H S E \\
(m e a n \pm S D)\end{array}$ & $\begin{array}{c}\text { Primary HSE } \\
(\text { mean } \pm S D)\end{array}$ & $\begin{array}{c}\text { Ex vivo skin } * \\
(\text { mean } \pm S D)\end{array}$ \\
\hline \multicolumn{5}{|c|}{ Group 1: TERT-HSE = ex vivo skin } \\
\hline CXCL-8/IL-8 & Proinflam; Ang; Epith & $22.655 \pm 12.774$ & $78.800 \pm 8.938^{\mathrm{b}, 2}$ & $19.647 \pm 13.512$ \\
\hline CCL-5/RANTES & Inflam & $108 \pm 16$ & $191 \pm 18^{\mathrm{b}, 1}$ & $83 \pm 40$ \\
\hline TIMP-2 & TR; Epith & $35.010 \pm 5.846$ & $53.889 \pm 8.778$ & $38.355 \pm 32.576$ \\
\hline $\mathrm{TNF}-\alpha$ & Proinflam & nd & nd & nd \\
\hline IL-1 $\alpha$ & Proinflam & nd & nd & $22 \pm 15$ \\
\hline \multicolumn{5}{|c|}{ Group 2: TERT-HSE > ex vivo skin } \\
\hline CXCL-1/GRO- $\alpha$ & Inflam; Ang; Epith & $35.439 \pm 17.009$ & $421.172 \pm 44.395^{\mathrm{d}, 4}$ & $3.109 \pm 1.689$ \\
\hline CCL-2/MCP-1 & Inflam; Ang; Epith; TR & $15.945 \pm 7.442$ & $69.153 \pm 12.190^{\mathrm{c}, 3}$ & $1.497 \pm 1.124$ \\
\hline \multicolumn{5}{|c|}{ Group 3: TERT-HSE < ex vivo skin } \\
\hline IL-6 & Proinflam; Gran; Ang & $568 \pm 315^{\mathrm{a}}$ & $3.638 \pm 873^{\mathrm{a}}$ & $131.756 \pm 64.057$ \\
\hline VEGF & Ang & nd & $522 \pm 41^{\mathrm{a}}$ & $2.425 \pm 499$ \\
\hline HGF & TR; Epith; Gran; Ang & nd & $2.330 \pm 203^{b}$ & $6.227 \pm 4.209$ \\
\hline
\end{tabular}

Statistical analysis: comparison of TERT-HSE or primary HSE with ex vivo skin; one-way ANOVA, Bonferroni's multiple comparison tests or unpaired $t$-test $\left({ }^{\mathrm{a}, 1} p<0.05 ;{ }^{\mathrm{b}, 2} p<0.01 ;{ }^{\mathrm{c}, 3} p<0.001 ;{ }^{\mathrm{d}, 4} p<0.0001 ;{ }^{\mathrm{a}-\mathrm{d}}\right.$ comparison between TERT-HSE or primary HSE and $e x$ vivo skin; ${ }^{1-4}$ comparison between TERT-HSE and primary HSE).

*Ex vivo skin data are adapted from Spiekstra et al. ${ }^{37}$

Ang, angiogenic; Epith, epithelialization; Gran, granulation tissue stimulating; HSE, human skin equivalent; Inflam, inflammatory chemokine for lymphocytes, neutrophils, and/or macrophages; nd: below detection limit; Proinflam, proinflammatory; TR, tissue remodeling. 

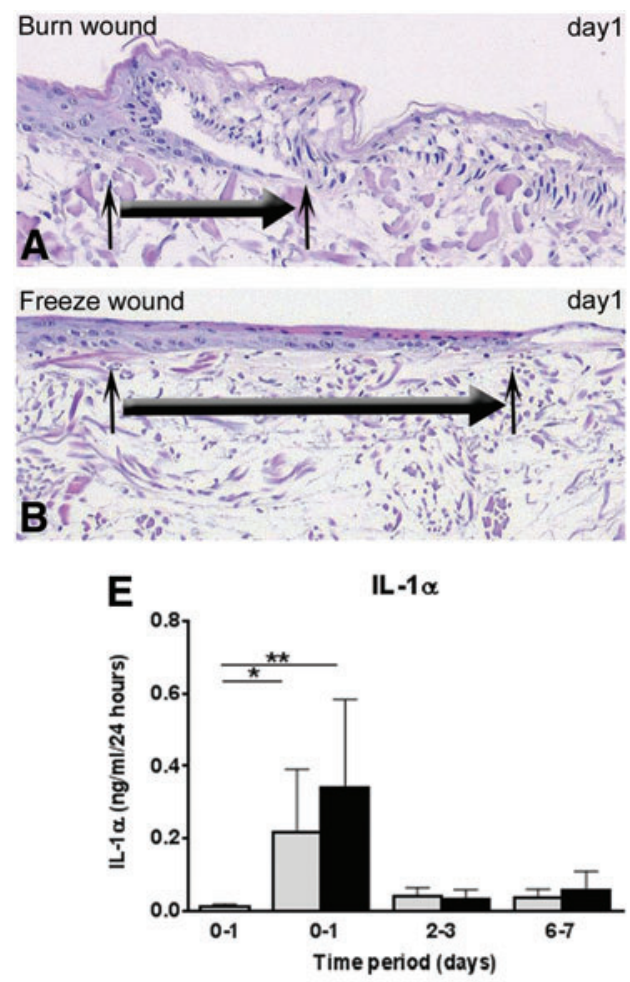

CCL-20
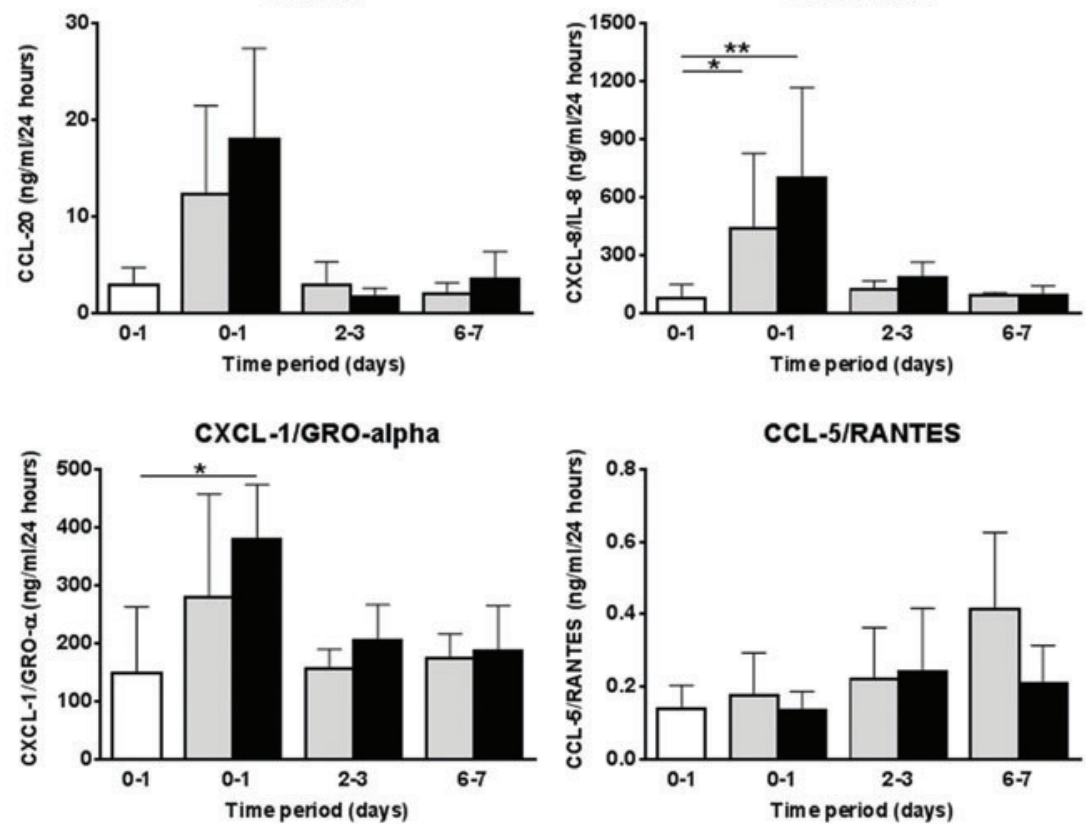

day7

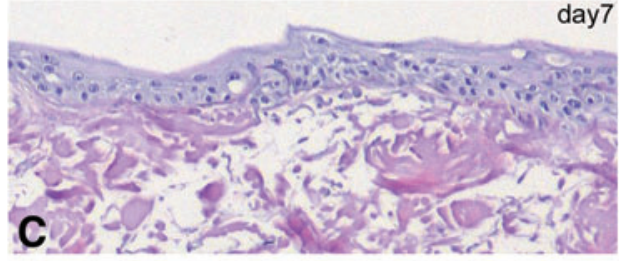

day7

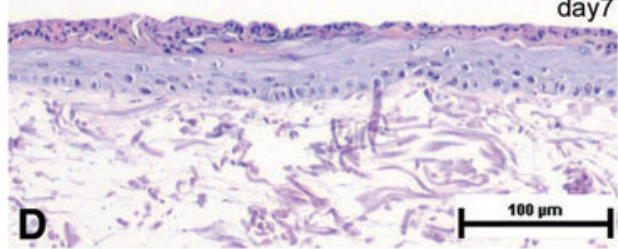

IL-6

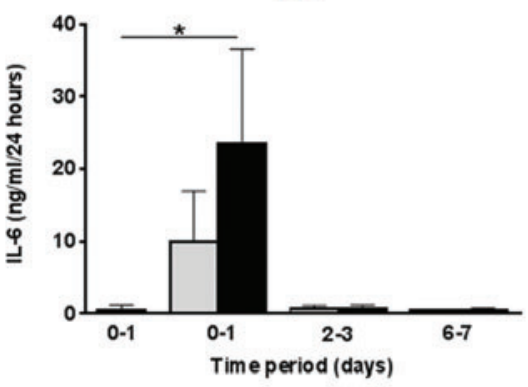

CXCL-8/LL-8

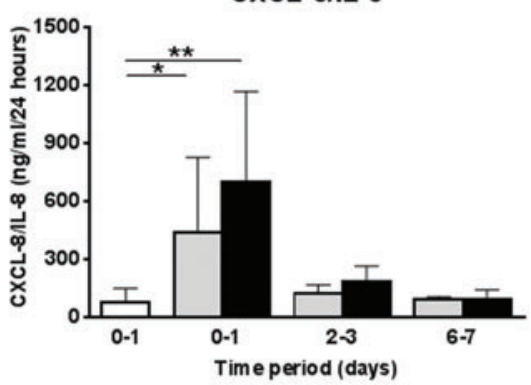

FIG. 4. Reepithelialization and protein secretion of wound-healing mediators after burn and cold injury in cell line SEs. Representative hematoxylin and eosin staining of epidermal ingrowth within TERT-HSEs after burn injury (A) and cold injury (B) 1 day after wounding. The large arrow represents the direction of the migrating epidermal front, and the small arrows indicate the start and the end of the epidermal ingrowth. Complete closure after burn (C) or cold (D) injury is shown 7 days after injury. Scale bar represents $100 \mu \mathrm{m}$. (E) Secretion profiles of (pro)inflammatory mediators IL- $1 \alpha$, IL-6, CCL-20, CXCL-8/IL-8, CXCL-1/GRO- $\alpha$, and CCL-5/RANTES after burn (gray bars) or cold (black bars) injury during time. White bar represents unwounded baseline secretion over $24 \mathrm{~h}$. Time period 0-1 represents supernatant harvested during the first $24 \mathrm{~h}$ after wounding, time periods 2-3 and 6-7 represent supernatant collected during $24 \mathrm{~h}$ from day 2 to day 3 or day 6 until day 7 after wounding, respectively. Each bar represents the mean \pm SD of four to five independent experiments. Statistical analysis: KruskalWallis test followed by Dunn's multiple comparisons test; * $p<0.05$; ** $p<0.01$.

Color images available online at www.liebertpub.com/tea
(Pro)inflammatory cytokine and chemokine secretion is related to the inflammatory phase of wound healing. ${ }^{59-61}$ Cytokines and chemokines (IL-1 $\alpha$, IL-6, CCL-20, CXCL$8 / \mathrm{IL}-8$, and CXCL-1/GRO- $\alpha$ ) showed a rapid increase during the first $24 \mathrm{~h}$ after burn or cold injury followed by a decrease toward homeostatic levels within the period thereafter (Fig. 4E). In contrast, the inflammatory chemokine CCL-5/RANTES secretion increased steadily during the 7-day study period after burn or cold injury (trend) within TERT-HSEs (Fig. 4E). No significant differences in cytokine or chemokine profiles were observed between burn and cold injury-inflicted wounds within TERT-HSEs (Fig. 4E).

Taken together, our results show that the TERT-HSE, based on TERT-immortalized keratinocytes and fibroblasts, not only closely resembles the morphology and proliferation rate of normal, native human skin but also secretes a mixture of cytokines and chemokines similar to native excised skin. In addition, the TERT-HSE is able to respond to environmental assault (wounding) in a similar way to primary $\mathrm{HSE}^{9}$ 
and most importantly to native skin, ${ }^{60}$ with regard to reepithelialization and secretion of inflammatory mediators.

\section{Discussion}

In this study, we successfully developed a functional full-thickness tissue-engineered SE entirely from human TERT-immortalized cell lines. The TERT-HSE consists of a well-differentiated epidermis on top of a fibroblast-populated dermis and closely resembles the morphology of native skin and primary HSE. The TERT-HSE has a normal proliferation rate within basal layer keratinocytes. The skin construct secretes a cocktail of cytokines and chemokines related to skin homeostasis and inflammation. Stress-related signs are absent during culturing of the construct, since the hyperproliferative marker, K6, is absent. The advantages of this well-defined cell line in vitro skin model above the use of freshly isolated primary skin cells are the continuous supply of easily amplified cells, no donor variation, and no logistical (transport from the clinic to laboratory) and ethical issues. With the effective proof-of-concept wound-healing model, we show that the TERT-HSE is functional. It has the potential to be an important tool to study the skin physiology and multiple other applications, and may contribute to the replacement, reduction, and refinement of animal models in the future.

To our knowledge, this is the first study that describes a fullthickness in vitro skin model, in which both the epidermal and dermal compartment are constructed entirely from immortalized cell lines. Previously, the TERT-immortalized keratinocyte cell line was used to construct a full-thickness SE, in which primary fibroblasts were seeded into the dermal compartment. ${ }^{39,40,50,53}$ In line with our model, the TERT-immortalized keratinocytes differentiated excellently. Other keratinocyte cell lines, which have been used for the construction of the epidermal component of a 3D-organotypic skin culture, are HaCaT-, NIKS-, HPV-16-, and HPV-18immortalized keratinocytes, Cdk4-overexpressing keratinocytes, and Y-27632 (Rho kinase inhibitor)-immortalized keratinocytes. However, the dermal counterpart of these constructs contained human dermal fibroblasts or $3 \mathrm{~T} 3$ feeder mouse fibroblasts. $41,42,44,46,48,49,51,52,62-66$ Unfortunately, the HaCaT HSEs showed deficiencies in epidermal differentiation and stratification depending on the culture conditions ${ }^{64,65}$ and the HPV HSEs displayed a disorganized epidermal layer. ${ }^{41}$

The barrier function is one of the main functions of human skin and an important feature, for example, drug targeting studies. It effectively protects against foreign substances/pathogens and prevents dehydration. The SC is a key component of the barrier structure. Our EM pictures showed a well-developed multilayered SC with corneosomes interconnecting the layers. Together with the findings of Van Drongelen et al., who showed that SEs made of TERT keratinocytes and primary fibroblasts exhibit a similar lipid organization and permeability as the SC of primary HSEs, and few differences in lipid composition, ${ }^{53}$ we suggest that the TERT-HSEs do have a barrier capacity that is mimicking the native skin. ${ }^{67}$

Desmosomes, which are located in the cell membrane, interconnect the keratinocytes. ${ }^{29}$ They provide strength to the skin to withstand movements. Malformation of desmosomes could lead to blister diseases. ${ }^{28,31,32}$ Our TERT-HSEs exhibit well-developed desmosomes within the SG, SS, and $\mathrm{SB}$, giving strength to the construct.

For the development of the BM, cross talk through paracrine signaling between the keratinocytes and fibroblasts is necessary. ${ }^{34,35,68}$ Our TERT-HSEs showed expression of the BM proteins, laminin 5 (lamina lucida) and collagen IV (lamina densa), which was located at the interface of the epidermis and the dermal collagen-elastin (bovine) matrix. This was confirmed by EM, where the presence of a lamina lucida and lamina densa was shown and in addition the presence of hemidesmosomes, which connect the epidermis to the ECM. Therefore, we can conclude that paracrine signaling between TERT-immortalized keratinocytes and fibroblasts occurs in our model, resulting in the construction of a well-developed BM with hemidesmosomes within 2 weeks. Since the BM is present in the TERT-HSEs, we expect to have a well-anchored epidermis to the dermis and a mechanical barrier, which is comparable to native skin.

Within the dermis, the TERT-HSEs exhibited the ECM markers, collagen III, and fibronectin, like native skin, whereas the primary construct lacked the collagen III protein. Thus, the TERT keratinocyte and fibroblast cross talk results in the production of collagen III, whereas primary keratinocytes and fibroblasts are not capable to produce collagen III. The observed amount of $\alpha$-SMA-positive cells within the TERT-HSEs is comparable to primary HSEs and the previous results of van den Broek et al. ${ }^{10}$ In conclusion, the TERTHSEs do not exhibit signs of hypertrophic scarring.

Overall, the TERT-HSE closely resembles the structure and composition of native skin.

For regulation of normal skin homeostasis and in response to environmental stimuli (danger), the secretion of cytokines, chemokines, and growth factors is essential. $9,37,60,61$ In this study, we showed that the secretome of the TERT-HSE consists of a cocktail of many cytokines and chemokines. Interestingly, the secretome of the TERT-HSE more closely resembles that of adult excised skin than primary HSE in general. However, the growth factors, VEGF and HGF, were not detectable in the supernatant of TERTHSEs, whereas they were secreted into the supernatant of $e x$ vivo skin and primary HSEs. This might be partly explained by the presence of endothelial cells and other cell types within the excised skin. However, TERT fibroblasts cultured in the absence of TERT keratinocytes did secrete low amounts of VEGF and HGF (data not shown), indicating that, possibly, uptake is occurring from the culture supernatant in the TERT-HSE model.

As a proof-of-principle for functionality, we demonstrated that the TERT-HSE provides a powerful tool to study wound healing in vitro. In native skin, two processes occur parallel after wounding, for example, secretion of multiple inflammatory mediators, which regulate infiltration of neutrophils, macrophages, and lymphocytes into the wound bed, and reepithelialization to close the wound. Upon wounding, our TERT-HSE showed an increase of a broad panel of inflammatory cytokines and chemokines within $24 \mathrm{~h}$ in response to cellular damage, mimicking the acute inflammatory phase of in vivo wound healing. ${ }^{60,61}$ This finding was similar to our earlier findings with primary in vitro HSE, except for CXCL-1/GRO- $\alpha$, which showed an increase directly after injury and remained elevated in primary HSEs. ${ }^{9}$ 
Burn injuries repair with more (hypertrophic) scar formation compared to cold injuries in vivo. In cold injuries, our cell line in vitro skin model equivalent showed a faster reepithelialization directly after wounding compared to burn wounds. A similar phenomenon was observed in the primary HSEs, where they hypothesized that within cold injuries the $\mathrm{BM}$ is still intact, whereas within a burn wound the BM proteins are denatured. ${ }^{9}$ Despite the morphological differences concerning the epithelial ingrowth at day 1 between the freeze and the burn wounds, no significant differences with respect to the secretome were determined between the different wound types in the cell line model, similar to the observations in the primary model. ${ }^{9}$ Thus, the TERT-HSE is able to produce wound-healing mediators, which are necessary for wound closure, and is able to restore the epidermis after different types of injury by cross talk through the TERT keratinocytes and fibroblasts.

In conclusion, in this study, we describe the first physiologically relevant full-thickness human SE constructed entirely from cell lines. The use of these cells enables scaling up for human SE production, creates less logistical hurdles, and eliminates the influence of donor variation. Therefore, this SE model provides an excellent opportunity to study in vitro skin biology and can also be used for drug targeting and testing new therapeutics and, ultimately, for incorporating into skin-on-a chip in the future. ${ }^{13}$

\section{Acknowledgments}

We thank G.F.H. Diercks, MD, PhD (University Medical Centre Groningen, Groningen, The Netherlands) for his professional help with the EM and G.C. Limandjaja, MD (VU University Medical Centre, Amsterdam, The Netherlands) for technical assistance. Part of this work has been performed at the Giepmans laboratory (University Medical Centre Groningen, Groningen, The Netherlands), which is sponsored by ZonMW (Grant 91111.006). This study was, in part, supported by the EuroTransBio grant (ETB09010) and, in part, by the Dutch Government ZonMW (MKMD Project No. 40-42600-98-010).

\section{Disclosure Statement}

Prof. S.G. and Prof. R.S. are cofounders of A-SKIN Netherland BV, which is a VUmc skin tissue engineering spin off company (SME).

\section{References}

1. Pappinen, S., Pryazhnikov, E., Khiroug, L., Ericson, M.B., Yliperttula, M., and Urtti, A. Organotypic cell cultures and two-photon imaging: tools for in vitro and in vivo assessment of percutaneous drug delivery and skin toxicity. $\mathrm{J}$ Control Release 161, 656, 2012.

2. Groeber, F., Holeiter, M., Hampel, M., Hinderer, S., and Schenke-Layland, K. Skin tissue engineering-in vivo and in vitro applications. Adv Drug Deliv Rev 63, 352, 2011.

3. Mathes, S.H., Ruffner, H., and Graf-Hausner, U. The use of skin models in drug development. Adv Drug Deliv Rev 69, 81, 2014.

4. Gibbs, S. In vitro irritation models and immune reactions. Skin Pharmacol Physiol 22, 103, 2009.

5. Gibbs, S., Corsini, E., Spiekstra, S.W., Galbiati, V., Fuchs, H.W., Degeorge, G., et al. An epidermal equivalent assay for identification and ranking potency of contact sensitizers. Toxicol Appl Pharmacol 15, 529, 2013.

6. Xie, Y., Rizzi, S.C., Dawson, R., Lynam, E., Richards, S., Leavesley, D.I., et al. Development of a three-dimensional human skin equivalent wound model for investigating novel wound healing therapies. Tissue Eng Part C Methods 16, 1111, 2010.

7. Coolen, N.A., Schouten, K.C., Boekema, B.K., Middelkoop, E., and Ulrich, M.M. Wound healing in a fetal, adult, and scar tissue model: a comparative study. Wound Repair Regen 18, 291, 2010.

8. MacNeil, S. Progress and opportunities for tissue-engineered skin. Nature 445, 874, 2007.

9. Breetveld, M., Richters, C.D., Rustemeyer, T., Scheper, R.J., and Gibbs, S. Comparison of wound closure after burn and cold injury in human skin equivalents. J Invest Dermatol 126, 1918, 2006.

10. van den Broek, L.J., Niessen, F.B., Scheper, R.J., and Gibbs, S. Development, validation and testing of a human tissue engineered hypertrophic scar model. ALTEX 29, 389, 2012.

11. Coolen, N.A., Vlig, M., van den Bogaerdt, A.J., Middelkoop, E., and Ulrich, M.M. Development of an in vitro burn wound model. Wound Repair Regen 16, 559, 2008.

12. Haisma, E.M., de, B.A., Chan, H., van Dissel, J.T., Drijfhout, J.W., Hiemstra, P.S., et al. LL-37-derived peptides eradicate multidrug-resistant Staphylococcus aureus from thermally wounded human skin equivalents. Antimicrob Agents Chemother 58, 4411, 2014.

13. van den Broek, L.J., Limandjaja, G.C., Niessen, F.B., and Gibbs, S. Human hypertrophic and keloid scar models: principles, limitations and future challenges from a tissue engineering perspective. Exp Dermatol 23, 382, 2014.

14. Shepherd, J., Douglas, I., Rimmer, S., Swanson, L., and MacNeil, S. Development of three-dimensional tissueengineered models of bacterial infected human skin wounds. Tissue Eng Part C Methods 15, 475, 2009.

15. Popov, L., Kovalski, J., Grandi, G., Bagnoli, F., and Amieva, M.R. Three-dimensional human skin models to understand Staphylococcus aureus skin colonization and infection. Front Immunol 5, 41, 2014.

16. Gibbs, S., van de Sandt, J.J., Merk, H.F., Lockley, D.J., Pendlington, R.U., and Pease, C.K. Xenobiotic metabolism in human skin and 3D human skin reconstructs: a review. Curr Drug Metab 8, 758, 2007.

17. Gotz, C., Pfeiffer, R., Tigges, J., Blatz, V., Jackh, C., Freytag, E.M., et al. Xenobiotic metabolism capacities of human skin in comparison with a 3D epidermis model and keratinocyte-based cell culture as in vitro alternatives for chemical testing: activating enzymes (Phase I). Exp Dermatol 21, 358, 2012.

18. Hewitt, N.J., Edwards, R.J., Fritsche, E., Goebel, C., Aeby, P., Scheel, J., et al. Use of human in vitro skin models for accurate and ethical risk assessment: metabolic considerations. Toxicol Sci 133, 209, 2013.

19. Lemper, M., Snykers, S., Vanhaecke, T., De, P.K., and Rogiers, V. Current status of healthy human skin models: can histone deacetylase inhibitors potentially improve the present replacement models? Skin Pharmacol Physiol 27, 36, 2014.

20. Cohen, C., Dossou, K.G., Rougier, A., and Roguet, R. Episkin: an in vitro model for the evaluation of phototoxicity and sunscreen photoprotective properties. Toxicol In Vitro 8, 669, 1994. 
21. Lelievre, D., Justine, P., Christiaens, F., Bonaventure, N., Coutet, J., Marrot, L., et al. The EpiSkin phototoxicity assay (EPA): development of an in vitro tiered strategy using 17 reference chemicals to predict phototoxic potency. Toxicol In Vitro 21, 977, 2007.

22. Gibbons, M.C., Foley, M.A., and Cardinal, K.O. Thinking inside the box: keeping tissue-engineered constructs in vitro for use as preclinical models. Tissue Eng Part B Rev 19, 14, 2013.

23. Gibbs, S., Silva Pinto, A.N., Murli, S., Huber, M., Hohl, D., and Ponec, M. Epidermal growth factor and keratinocyte growth factor differentially regulate epidermal migration, growth, and differentiation. Wound Repair Regen 8, 192, 2000.

24. Ponec, M. Reconstruction of human epidermis on deepidermized dermis: expression of differentiation-specific protein markers and lipid composition. Toxicol In Vitro 5, 597, 1991.

25. Regnier, M., Prunieras, M., and Woodley, D. Growth and differentiation of adult human epidermal cells on dermal substrates. Front Matrix Biol 9, 4, 1981.

26. Bell, E., Paul Ehrlich, H., Buttle, D., and Nakatsuij, T. Living tissue formed in vitro and accepted as skin-equivalent tissue of full thickness. Science 211, 1052, 1981.

27. Navarro, J.M., Casatorres, J., and Jorcano, J.L. Elements controlling the expression and induction of the skin hyperproliferation-associated keratin K6. J Biol Chem 270, 21362, 1995.

28. Korver, J.E., van Duijnhoven, M.W., Pasch, M.C., van Erp, P.E., and van de Kerkhof, P.C. Assessment of epidermal subpopulations and proliferation in healthy skin, symptomless and lesional skin of spreading psoriasis. $\mathrm{Br} \mathrm{J}$ Dermatol 155, 688, 2006.

29. Fuchs, E. Epidermal differentiation: the bare essentials. J Cell Biol 111, 2807, 1990.

30. Corcuff, P., Fiat, F., and Minondo, A.M. Ultrastructure of the human stratum corneum. Skin Pharmacol Appl Skin Physiol 14 (Suppl 1), 4, 2001.

31. van der Wier, G., Jonkman, M.F., Pas, H.H., and Diercks, G.F. Ultrastructure of acantholysis in pemphigus foliaceus re-examined from the current perspective. Br J Dermatol 167, 1265, 2012.

32. Kitajima, Y. New insights into desmosome regulation and pemphigus blistering as a desmosome-remodeling disease. Kaohsiung J Med Sci 29, 1, 2013.

33. van der Wier, G., Pas, H.H., Kramer, D., Diercks, G.F., and Jonkman, M.F. Smaller desmosomes are seen in the skin of pemphigus patients with anti-desmoglein 1 antibodies but not in patients with anti-desmoglein 3 antibodies. J Invest Dermatol 134, 2287, 2014.

34. Breitkreutz, D., Koxholt, I., Thiemann, K., and Nischt, R. Skin basement membrane: the foundation of epidermal integrity-BM functions and diverse roles of bridging molecules nidogen and perlecan. Biomed Res Int 2013, 179784, 2013.

35. El Ghalbzouri, A., Gibbs, S., Lamme, E., Van Blitterswijk, C.A., and Ponec, M. Effect of fibroblasts on epidermal regeneration. Br J Dermatol 147, 230, 2002.

36. El Ghalbzouri A., and Ponec, M. Diffusible factors released by fibroblasts support epidermal morphogenesis and deposition of basement membrane components. Wound Repair Regen 12, 359, 2004.

37. Spiekstra, S.W., Breetveld, M., Rustemeyer, T., Scheper, R.J., and Gibbs, S. Wound-healing factors secreted by epidermal keratinocytes and dermal fibroblasts in skin substitutes. Wound Repair Regen 15, 708, 2007.

38. Kroeze, K.L., Boink, M.A., Sampat-Sardjoepersad, S.C., Waaijman, T., Scheper, R.J., and Gibbs, S. Autocrine regulation of re-epithelialization after wounding by chemokine receptors CCR1, CCR10, CXCR1, CXCR2, and CXCR3. J Invest Dermatol 132, 216, 2012.

39. van Drongelen, V., Alloul-Ramdhani, M., Danso, M.O., Mieremet, A., Mulder, A., van, S.J., et al. Knock-down of filaggrin does not affect lipid organization and composition in stratum corneum of reconstructed human skin equivalents. Exp Dermatol 22, 807, 2013.

40. Vaughan, M.B., Ramirez, R.D., Andrews, C.M., Wright, W.E., and Shay, J.W. H-ras expression in immortalized keratinocytes produces an invasive epithelium in cultured skin equivalents. PLoS One 4, e7908, 2009.

41. Blanton, R.A., Perez-Reyes, N., Merrick, D.T., and McDougall, J.K. Epithelial cells immortalized by human papillomaviruses have premalignant characteristics in organotypic culture. Am J Pathol 138, 673, 1991.

42. Slavik, M.A., Allen-Hoffmann, B.L., Liu, B.Y., and Alexander, C.M. Wnt signaling induces differentiation of progenitor cells in organotypic keratinocyte cultures. BMC Dev Biol 7, 9, 2007.

43. Commandeur, S., van Drongelen V, de Gruijl, F.R., and El, G.A. Epidermal growth factor receptor activation and inhibition in $3 \mathrm{D}$ in vitro models of normal skin and human cutaneous squamous cell carcinoma. Cancer Sci 103, 2120, 2012.

44. Vaughan, M.B., Ramirez, R.D., Brown, S.A., Yang, J.C., Wright, W.E., and Shay, J.W. A reproducible laser-wounded skin equivalent model to study the effects of aging in vitro. Rejuvenation Res 7, 99, 2004.

45. Commandeur, S., de Gruijl, F.R., Willemze, R., Tensen, C.P., and El, G.A. An in vitro three-dimensional model of primary human cutaneous squamous cell carcinoma. Exp Dermatol 18, 849, 2009.

46. Schoop, V.M., Mirancea, N., and Fusenig, N.E. Epidermal organization and differentiation of HaCaT keratinocytes in organotypic coculture with human dermal fibroblasts. J Invest Dermatol 112, 343, 1999.

47. Okugawa, Y., and Hirai, Y. Overexpression of extracellular epimorphin leads to impaired epidermal differentiation in HaCaT keratinocytes. J Invest Dermatol 128, 1884, 2008.

48. Allen-Hoffmann, B.L., Schlosser, S.J., Ivarie, C.A., Sattler, C.A., Meisner, L.F., and O'Connor, S.L. Normal growth and differentiation in a spontaneously immortalized neardiploid human keratinocyte cell line, NIKS. J Invest Dermatol 114, 444, 2000.

49. Gibson, A.L., Thomas-Virnig, C.L., Centanni, J.M., Schlosser, S.J., Johnston, C.E., Van Winkle, K.F., et al. Nonviral human beta defensin-3 expression in a bioengineered human skin tissue: a therapeutic alternative for infected wounds. Wound Repair Regen 20, 414, 2012.

50. Dickson, M.A., Hahn, W.C., Ino, Y., Ronfard, V., Wu, J.Y., Weinberg, R.A., et al. Human keratinocytes that express hTERT and also bypass a p16(INK4a)-enforced mechanism that limits life span become immortal yet retain normal growth and differentiation characteristics. Mol Cell Biol 20, 1436, 2000.

51. Chapman, S., Liu, X., Meyers, C., Schlegel, R., and McBride, A.A. Human keratinocytes are efficiently immortalized by a Rho kinase inhibitor. J Clin Invest $\mathbf{1 2 0}$, 2619, 2010. 
52. van den Bogaard, E.H., Rodijk-Olthuis, D., Jansen, P.A., van Vlijmen-Willems, I.M., van Erp, P.E., Joosten, I., et al. Rho kinase inhibitor Y-27632 prolongs the life span of adult human keratinocytes, enhances skin equivalent development, and facilitates lentiviral transduction. Tissue Eng Part A 18, 1827, 2012.

53. van Drongelen, V., Danso, M.O., Mulder, A., Mieremet, A., van, S.J., Bouwstra, J.A., et al. Barrier properties of an N/ TERT based human skin equivalent. Tissue Eng Part A 20, 3041, 2014.

54. Shay, J.W., and Wright, W.E. Use of telomerase to create bioengineered tissues. Ann N Y Acad Sci 1057, 479, 2005.

55. Lee, K.M., Choi, K.H., and Ouellette, M.M. Use of exogenous hTERT to immortalize primary human cells. Cytotechnology 45, 33, 2004.

56. Bodnar, A.G., Ouellette, M., Frolkis, M., Holt, S.E., Chiu, C.P., Morin, G.B., Harley C.B., Shay, J.W., Lichtsteiner, S., and Wright, W.E. Extension of life-span by introduction of telomerase into normal human cells. Science 279, 349, 1998.

57. Waaijman, T., Breetveld, M., Ulrich, M., Middelkoop, E., Scheper, R.J., and Gibbs, S. Use of a collagen-elastin matrix as transport carrier system to transfer proliferating epidermal cells to human dermis in vitro. Cell Transplant 19, 1339, 2010.

58. Kroeze, K.L., Jurgens, W.J., Doulabi, B.Z., van Milligen, F.J., Scheper, R.J., and Gibbs, S. Chemokine-mediated migration of skin-derived stem cells: predominant role for CCL5/RANTES. J Invest Dermatol 129, 1569, 2009.

59. Goldman, R. Growth factors and chronic wound healing: past, present, and future. Adv Skin Wound Care 17, 24, 2004.

60. Werner, S., and Grose, R. Regulation of wound healing by growth factors and cytokines. Physiol Rev 83, 835, 2003.

61. Broughton, G., Janis, J.E., and Attinger, C.E. The basic science of wound healing. Plast Reconstr Surg 117, 12S, 2006.

62. Stark, H.J., Szabowski, A., Fusenig, N.E., and MaasSzabowski, N. Organotypic cocultures as skin equivalents: a complex and sophisticated in vitro system. Biol Proced Online 6, 55, 2004.
63. Rasmussen, C., Gratz, K., Liebel, F., Southall, M., Garay, M., Bhattacharyya, S., et al. The StrataTest(R) human skin model, a consistent in vitro alternative for toxicological testing. Toxicol In Vitro 24, 2021, 2010.

64. Zanoni, T.B., Tiago, M., Faiao-Flores, F., de Moraes Barros, S.B., Bast, A., Hageman, G., et al. Basic Red 51, a permitted semi-permanent hair dye, is cytotoxic to human skin cells: studies in monolayer and 3D skin model using human keratinocytes (HaCaT). Toxicol Lett 227, 139, 2014.

65. Maas-Szabowski, N., Starker, A., and Fusenig, N.E. Epidermal tissue regeneration and stromal interaction in $\mathrm{HaCaT}$ cells is initiated by TGF-alpha. J Cell Sci 116, 2937, 2003.

66. Boelsma, E., Verhoeven, M.C., and Ponec, M. Reconstruction of a human skin equivalent using a spontaneously transformed keratinocyte cell line (HaCaT). J Invest Dermatol 112, 489, 1999.

67. Ponec, M., Gibbs, S., Pilgram, G., Boelsma, E., Koerten, H., Bouwstra, J., et al. Barrier function in reconstructed epidermis and its resemblance to native human skin. Skin Pharmacol Appl Skin Physiol 14 (Suppl 1), 63, 2001.

68. El Ghalbzouri A., Lamme, E., and Ponec, M. Crucial role of fibroblasts in regulating epidermal morphogenesis. Cell Tissue Res 310, 189, 2002.

Address correspondence to: Susan Gibbs, PhD Department of Oral Cell Biology Academic Centre for Dentistry Amsterdam (ACTA) University of Amsterdam and VU University Amsterdam Room 11 N69

Gustav Mahlerlaan 3004 1081 LA Amsterdam The Netherlands

E-mail: s.gibbs@acta.nl

Received: March 24, 2015

Accepted: June 26, 2015

Online Publication Date: August 3, 2015 